\title{
LOS MODELOS DE ORIENTACIÓN EN ESPAÑA. PRESENTE Y FUTURO
}

\author{
COUNSELLING MODELS IN SPAIN. PRESENT AND FUTURE
}

\author{
Manuel Álvarez González* \\ Universidad de Barcelona
}

\begin{abstract}
RESUMEN
El propósito de este trabajo es llevar a cabo un análisis de los modelos de orientación en España, concretamente en las diferentes Comunidades Autónomas. A partir de ahí, efectuar unas propuestas de mejora, que han de ir acompañadas de una nueva estructura organizativa que complemente la actual. Es decir, la necesidad de consensuar una serie de medidas de tipo legal que faciliten la operativización de la propuesta organizativa de la orientación.
\end{abstract}

Palabras clave: Modelos de orientación, niveles de intervención, nueva estructura organizativa de la orientación.

\begin{abstract}
The main purpose of this labour is to carry out an analysis of the guidance systems or models in Spain, specifically to the different Autonomous Communities. Starting from there, make some improvement proposals, wich ought to be accompanied by a new organization structure which completes the present one. It means, the need to assent a number of legal type measures which make easy the functionality of the organization proposal of the counseling.
\end{abstract}

Key words: Guidance models, intervention levels, new organization structure of the counselin.

\section{Introducción}

En este estudio se pretende efectuar un análisis y una prospectiva de los modelos de Orientación en España, haciendo referencia a la práctica orientadora presente y futura.

\footnotetext{
* Manuel Álvarez González, es Profesor Titular del Departamento de Métodos de Investigación y Diagnóstico en Educación, de la Facultad de Ciencias de la Educación. Universidad de Barcelona. Actividad investigadora actual: Educación emocional y análisis de la trayectoria académica de los alumnos de bachillerato-universidad. Es coautor de numerosas publicaciones, y premio nacional de investigación e innovación educativa (cide, Madrid, 1989).
} 
Después de algo más de una década de implantación del modelo de orientación propuesto por la LOGSE con sus variantes en las diferentes Comunidades Autonómicas y en los umbrales de una nueva Ley de Educación (LOCE), ha llegado el momento de efectuar un análisis del mismo. Para ello, se hace una reflexión sobre el desarrollo institucional de la orientación en nuestro país, se efectúa un análisis del modelo de orientación en las distintas Comunidades Autónomas y, una vez efectuado ese análisis, se sugieren una serie de propuestas de mejora y se plantean un conjunto de presupuestos para la operativización de la orientación en España. Para dar respuesta a la nueva realidad (multiculturalismo, avances tecnológicos, ampliación de la escolaridad, ayuda a colectivos desfavorecidos, etc.) y subsanar los déficits de la actual práctica orientadora se plantea una nueva estructura organizativa que complemente la actual.

La finalidad de este trabajo es llevar a cabo una reflexión del modelo/s de orientación en España que nos permita plantear algunas alternativas de mejora, en un momento de cambio de Sistema Educativo y después de más de una década de implantación de dicho modelo.

\section{Desarrollo institucional de la orientación}

Para poder analizar adecuadamente el modelo actual de la orientación en España es conveniente hacer un breve repaso a lo que ha sido hasta estos momentos el desarrollo institucional de la orientación en nuestro país. Son muchos los expertos en orientación que han efectuado estudios, con un gran rigor documental, sobre este proceso de institucionalización de la orientación (Lázaro y otros, 1982; Díaz Allué, 1989; Álvarez González, 1995; Bisquerra, 1996; Benavent, 1996, 2000; Sobrado y otros, 1998...).

En este trabajo se intenta recuperar algunos aspectos que pueden considerarse de interés:

- Hasta 1970 la característica que define el desarrollo de la orientación en España se concreta en una abundante legislación sobre la orientación, pero con escasa concreción en la práctica, debido al poco convencimiento de su utilidad por parte de los gobernantes y a la escasez de recursos (Lázaro y otros, 1982). La orientación no era sentida como una verdadera necesidad, sino más bien como una imposición administrativa (García Yagüe, 1978).

- Será a partir de 1970 con la implantación de la Ley General de Educación (LGE) del 70 cuando se comienza a dar una dimensión educativa a la orientación. Se produce un relanzamiento de los Servicios de Orientación Escolar y Vocacional (SOEV) con tareas de orientación académica y profesional y con una estructura provincial y en 1980 con la implantación de los Equipos Multiprofesionales con funciones de Educación Especial. Destacar, que, en algunos municipios, se constituyeron los Equipos SocioPsicopedagógicos Municipales como complemento a los servicios estatales y posteriormente a los autonómicos.

- En la década de los 80 tiene lugar la gran proliferación y consolidación de los servicios de orientación. Concretamente, a partir de 1983 se crean los servicios autonómicos en aquellas Comunidades con competencias en Educación (Cataluña, 1983; Andalucía, 1983; País Vasco, 1984; Valencia, 1985; Galicia, 1985 y Canarias, 1986). Los diferentes servicios de orientación que actuaban en la comunidad educativa (SOEV, 
EM, etc.) se integran en los nuevos servicios de orientación autonómicos. Estos servicios presentan estructuras organizativas y funcionales muy similares en cuanto a ámbitos de actuación, modelos y áreas de intervención y cierta interdisciplinariedad. En el período 1970-1990 no se habla explícitamente de modelo organizativo, se hace alguna mención a la acción tutorial,

- Será en la década de los 90 con la implantación de la Ley Orgánica General del Sistema Educativo (LOGSE) cuando toma carta de naturaleza el modelo de la orientación. Se hace una verdadera propuesta de modelo organizativo y funcional de la orientación. Con el fin de dar respuesta a las nuevas necesidades educativas y de la orientación, la Administración educativa propone un modelo organizativo y funcional de la orientación, basado en tres niveles de intervención:

a) la acción tutorial como unidad de acción directa;

b) el Departamento de Orientación como unidad organizativa básica en el centro escolar (servicio interno) y

c) los equipos de sector como unidad eje en un área o sector escolar (servicio externo).

La orientación pasa a ser un elemento fundamental en el proceso educativo y, en consecuencia, en la actividad docente. El marco legal del modelo de orientación queda regulado en la LOGSE (art: 55 y 60). Una serie de documentos (normativas) han ayudado a desarrollar este modelo y su implantación: Proyecto de Reforma para la Enseñanza (1987); Libro Blanco para la Reforma del sistema Educativo (1989); Orientación Educativa e Intervención Psicopedagógica (1990); Orientación y tutoría (1992); Estructura y funciones de los Equipos de orientación educativa y psicopedagogía (1992); Instrucciones sobre el plan de actividades de los D.O. en Secundaria (1993), entre otros.

- En cuanto a la Ley de calidad (2002) hemos de señalar que la orientación no es considerada como un factor de calidad, apenas se hace alusión al modelo de orientación. Una pregunta queda en el aire: ¿Qué va a pasar con el modelo organizativo y funcional de la Orientación? Si bien en la LOGSE la Orientación tenía un carácter integrador, comprensivo y continuo (La orientación como una función total de la educación), en la LOCE tiene más bien un carácter segregador, adoptando el modelo de distribución y ajuste del alumnado en las materias, en los grupos, en los itinerarios, etc., en función de sus características personales (adaptar, controlar, dirigir o tomar responsabilidades). Se asume, especialmente, en los momentos de transición académica (momento crítico) con una función predominantemente diagnóstica. Hemos de ser conscientes que el cambio de sistema educativo, comporta un cambio de roles y funciones de la orientación.

Se espera que las nuevas reformas que se están haciendo de la Ley se vuelva a contemplar la orientación como una pieza básica de la calidad de la educación y se consolide el modelo de orientación que se propuso en la LOGSE.

En síntesis, diríamos que, en esa aproximación de la Orientación al marco educativo, dos han sido los modelos adoptados en nuestro país (Álvarez González, 1994, 1998):

- Antes de la implantación de la LOGSE la intervención orientadora se centró prioritariamente en la distribución y ajuste del alumnado al Sistema Educativo, Para ello, se 
utilizó el modelo de Servicios basado en el especialista. Esto no favoreció la implantación del profesional de la educación. Los especialistas, con el fin de afianzar su status, no compartieron dicha actividad con el profesorado/tutor.

- A partir de la LOGSE, se considera la orientación como una función total de la educación. Orientar ya no es adaptar, controlar, dirigir o tomar responsabilidades por otros, sino ofrecer al alumnado la ayuda necesaria para que comprendan, organicen, apliquen y desarrollen sus actividades. Aparece por primera vez el proceso orientador indisolublemente unido al proceso educativo. Es decir, las funciones y objetivos de la educación quedan asumidas en las funciones y objetivos de la educación. El grupo docente se convierte en el agente natural de la orientación y el programa de estudios constituye la vía natural a través de la cual se afrontan los objetivos de la orientación.

- Con la LOCE la orientación va a volver a adoptar la función de diagnóstico, distribución y ajuste del alumnado, si la nueva reforma de la Ley no lo remedia.

\section{Los sistemas de orientación en España. ¿Modelo o modelos de orientación?}

En este apartado se efectúa un análisis del modelo/s de orientación en España, posteriormente se plantean algunas propuestas de mejor y se termina sugiriendo una serie de presupuestos para una mejor operativización de la propuesta organizativa de la orientación.

\section{Análisis del modelo/s de Orientación en las diferentes Comunidades Autónomas}

En estos momentos, todas las Comunidades Autónomas (CC.AA.) tienen plenas transferencias en Educación, en consecuencia, pueden regular la orientación en su Comunidad. Ha llegado el momento que nos hagamos la siguiente pregunta: ¿Se ha de hablar de un modelo único de orientación con algunas variantes, o bien de diferentes modelos en las distintas CC.AA.?

Para el análisis de estos modelos de orientación nos vamos a servir de tres aspectos que son básicos:

a) El marco legal que viene representado por los documentos oficiales que ha generado el MEC y posteriormente las diferentes Comunidades Autónomas;

b) los teóricos de la orientación que han llevado a cabo estudios comparativos de los sistemas de orientación de las diferentes CC.AA. (Sobrado y otros, 1998; Sobrado, 1999; Carrión y Fernández, 1999; Sanz, 1999 y 2001; Rodríguez Moreno, 2002, entre otros) y

c) cómo se está afrontando la práctica orientadora.

De este análisis tridimensional podemos extraer las siguientes conclusiones:

a) La organización de los modelos de orientación responden a un único modelo, presentan estructuras administrativas muy similares a excepción de Cataluña que no contempla el Departamento de Orientación, pero sí los tres niveles de intervención con la presencia del profesor de psicología y pedagogía en Secundaria. 
b) Todas las Comunidades Autónomas, a excepción de Galicia, Navarra y Canarias (ésta sólo a nivel de normativa) han "adulterado" el modelo de orientación original, si tenemos en cuenta el mandato legal, porque no contemplan los tres niveles de intervención en la Educación Infantil y Primaria, como así constaba en los documentos de la Reforma. La estructura más generalizada no respeta el modelo inicial de los tres niveles de intervención.

c) Las Comunidades Autónomas, que últimamente han asumido competencias plenas en educación, no han tenido la "valentía" de contemplar el modelo de orientación con sus tres niveles de intervención en Infantil, Primaria y Secundaria. No obstante se está produciendo una cierta tendencia a reforzar los servicios internos (Departamento de orientación), como consecuencia de la evidente y paulatina aceptación de estos servicios en la institución educativa. Los centros de Primaria que han gozado de equipo de orientación dentro de la institución han valorado muy positivamente este apoyo interno para la dinámica tutorial y orientadora. Al mismo tiempo, comienzan a oírse voces autorizadas que insisten en la conveniencia de implantar el Departamento de Orientación en Educación Infantil y Primaria. Hemos de pensar que la decisión de la Administración de no contemplar el Departamento de Orientación en estos niveles educativos se debe más a aspectos presupuestarios que a una valoración negativa de la experiencia.

d) Si a nivel de estructura organizativa el modelo de orientación aparece perfectamente definido, no podemos decir lo mismo a nivel funcional. Las funciones no se presentan lo suficientemente explicitadas para cada uno de los tres niveles de intervención (Álvarez González, 1994, 1995; Benavent y Fossati, 1998a, 1998b; Díaz allué y otros, 1997; Rodríguez Espinar, 1995, 1998; Rodríguez Moreno, 1998; Sanz, 1995, 1999; Sobrado y otros, 1998; Sanz y sobrado, 1998). En la mayoría de las ocasiones, las funciones asignadas a los tres niveles de intervención no se corresponden con la composición de los mismos. Las diferencias que se aprecian en los modelos de las CC.AA. no están tanto en las funciones realizadas, sino en el nivel en que se afrontan cada una de ellas. Depende del número de profesionales implicados, del tiempo dedicado y del grado de cualificación que tengan. Una cosa es cierta, gran parte de las funciones del Equipo externo se solapan con el Departamento de Orientación.

e) Los servicios externos no tienen la suficiente interdisciplinariedad para asumir las funciones asignadas. Algunas CC.AA. (Galicia, Andalucía, Cataluña, Valencia, Canarias), contemplan la posibilidad de crear equipos de apoyo externo de carácter especializado de ámbito provincial, también denominados Equipos Específicos para la ayuda del alumnado con deficiencias sensoriales y motrices, con trastornos graves de desarrollo y con deficiencia visual.

f) La compleja composición de los Departamentos de Orientación hace dificil una acción conjunta. Aparecen poco explicitadas las funciones de sus componentes, especialmente el profesorado de apoyo a los ámbitos. El perfil de los componentes del Departamento de Orientación no está plenamente definido. Se ha de hacer la distinción:

1) Los que tienen competencias más específicas de orientación (profesor de psicología y pedagogía); 
2) Los que desarrollan las actividades de orientación en el marco de contextos, no especializados (profesor-tutor, profesor de FOL, maestro de pedagogía terapéutica). La composición suele variar ligeramente en las diferentes CC.AA.

g) Escasa dinamización y potenciación de la tutoría, debido fundamentalmente a la falta de actitud, información, formación del profesorado por la tarea tutorial y de una infraestructura adecuada. A esto hemos de añadir la poca definición del modelo tutorial.

h) Modelo de intervención. Analizada la normativa legal de la orientación de cada una de las CC.AA., no existen referencias explícitas a modelos de intervención a excepción de Andalucía. Si nos centramos en las funciones asignadas, el modelo predominante es el de intervención directa individual, con una implantación menor del modelo de programas y con un empezar a ver la necesidad del modelo de consulta. No obstante, resulta curioso que la Administración educativa recomiende la intervención por programas y en cambio el mandato legal señala explícitamente las funciones a desarrollar. Si se quiere afrontar una intervención por programas se han de flexibilizar las funciones propuestas.

i) En cuanto al modelo teórico hay una tendencia en las diferentes CC.AA. a pasar de un modelo clínico, basado en el diagnóstico a un modelo más psicopedagógico con una mayor implicación en los centros. Ninguna de las Comunidades asume, de forma plena, el modelo socio-psicopedagógico, no sólo centrado en el ámbito escolar, sino en el contexto social.

j) La coordinación, es una asignatura pendiente en todas las CC.AA. Se está haciendo una intervención bastante fragmentaria y con una cierta discontinuidad a nivel de:

1) Comunicación, cooperación y coordinación entre los diferentes servicios;

2) Intercambio de experiencias;

3) Integración de los distintos servicios. Algunas CC.AA. han intentado dar el primer paso, concentrando a nivel físico los distintos servicios educativos (Cataluña, Andalucía, etc.).

k) Las vías para el desarrollo y consolidación del modelo de orientación son tres: la jerárquica, la supervisión y formación y las asociaciones profesionales. En cuanto a la jerarquía o dependencias administrativas está bastante definida en las diferentes Comunidades. No podemos decir lo mismo cuando nos referimos a la supervisión y formación: No existen unos planes estructurados de formación continuada. No se dispone de un modelo integrador de formación para todos los implicados en la acción orientadora, ni el modelo de orientación está plenamente definido. Y en cuanto a la profesionalización decir que, en la mayoría de las CC.AA., los profesionales de la orientación no pertenecen a asociaciones profesionales. Las que existen tienen muy escasa implantación nacional (Cataluña, ACOEP; el resto de Comunidades, AEOP) o bien a nivel regional o provincial.

Podemos concluir que estamos en una fase de clarificación, implantación y ajuste de este modelo. Habrá de pasar aún más tiempo para llegar a su plena implantación y consolidación. En este proceso de adecuación y consolidación el nivel de intervención que está experimentando un mayor desarrollo es el Departamento de orientación (sólo en Secundaria, a excepción de Galicia y Navarra) como soporte técnico a la acción psicopedagógica. Las di- 
ferentes Administraciones educativas, a excepción de Cataluña, han considerado de obligada necesidad el priorizar este nivel, porque se ha de convertir, de hecho ya lo está haciendo, en el dinamizador de la acción tutorial y orientadora en el centro educativo. No podemos decir lo mismo de los otros dos niveles de intervención (tutoría y servicios de apoyo externo), cuyo proceso de reconversión y de adaptación va más lento. Hay autores que para definir la situación presente han utilizado expresiones como: Piedras en el camino (Santos, 1995); Luces y sombras (Rodríguez Espinar, 1995); Puntos oscuros (síntesis de las 1as Jornadas de orientación UNED-MEC, Madrid 1997); Dilemas o problemas (Tarrego, 1998); Incumplimientos (Álvarez González, 1998a); Tensiones (Coll, 1998); Conflictos (Carrión y Fernández, 1999); Dificultades y tensiones (Martín, 1999); Insatisfacción (Mateo, Álvarez González, Casanovas, Hernando, Sans, 2002).

\section{Propuestas de mejora}

Las diferentes propuestas podríamos agruparlas en torno a cuatro dimensiones:

\section{Clarificación y desarrollo del modelo organizativo y funcional de la orientación y la acción tutorial en los centros educativos}

Las Administraciones educativas de las diferentes Comunidades Autónomas han de desarrollar, de forma plena, un modelo organizativo y funcional de la orientación basado en tres niveles de intervención: Tutoría, Dpto. de orientación y Equipos de apoyo externo, teniendo en cuenta las peculiaridades de cada autonomía. Para ello, se han de delimitar claramente las tareas específicas para los tres niveles, con el fin de evitar suplantaciones y solapamientos y, al mismo tiempo, se ha de estimular una efectiva coordinación entre dichos niveles de intervención y éstos con el resto de servicios del contexto. Con ello, se operativizarían los recursos, se evitarían duplicidades y se presentaría una oferta unitaria y coherente. Del Valle (1987:20) sugiere la siguiente regla que puede ser perfectamente asumida por los servicios educativos y de orientación:" Lo que se pueda hacer en el nivel más próximo a las necesidades, no se haga desde el nivel o estancia más alejada".

Es necesario una reestructuración de los equipos de apoyo externo (EOE, EOEPS, SPE, EPA, etc.)

Estos equipos han de desarrollar tareas que complementen las del tutor/a y del psicopedagogo/a en el centro. Para ello, han de disponer de una mayor especialización e interdisciplinariedad, sus funciones se han de orientar en una doble dirección:

1) A nivel de centro, colaborando y asesorando al tutor y al psicopedadogo/a en aspectos curriculares y de orientación;

2) a nivel de comunidad, proporcionando la coordinación de los distintos recursos educativos y de orientación (Servicios Sociales, Sanitarios, Jurídicos, socio-comunitarios, Justicia y ocupación) de la zona, a través del intercambio de experiencias, grupos de trabajo, formación de los implicados en tareas de orientación, etc. Sus funcio- 
nes no han de tener solamente un carácter terapéutico y puntual, sino también preventivo y comprensivo.

La composición de estos equipos habrá de estar en relación con las funciones asignadas. Un ejemplo de lo que debería ser lo tenemos en la Comunidad Autónoma de Galicia que hace una apuesta por potenciar los servicios internos (Departamento de orientación) en los centros públicos de Infantil, Primaria y Secundaria y una reducción de los Equipos psicopedagógicos de Apoyo (1 por provincia) con funciones muy específicas de apoyo a los servicios internos.

\section{Implantación de los Departamentos de Orientación en los Centros de Infantil y Primaria y Secundaria}

Si queremos que la orientación tenga un carácter preventivo y de desarrollo $\mathrm{y}$, al mismo tiempo, la consideremos como un factor de calidad de la educación, las Administraciones han de dar el paso de su implantación y consolidación en estas etapas educativas; con ello, desarrollaríamos plenamente el modelo de orientación en nuestro país. Las funciones han de estar referidas al centro, al alumnado, al profesorado, familias y otras instituciones y han de tener un carácter prioritariamente preventivo, de desarrollo y de intervención social. Estas funciones se podrían agrupar en torno a seis grandes ámbitos:

1) Soporte a la organización y dinámica del centro (colaboración con el equipo directivo, consejo escolar, profesorado, familias, etc.);

2) apoyo al proceso de E-A. Atención a la diversidad (intervención directa o compartida en medidas preventivas ordinaria o específicas de atención a la diversidad);

3) orientación académica y profesional (ayuda y asesoramiento en los procesos de transición académica y socio-laboral);

4) plan de acción tutorial (colaboración en el diseño y elaboración del PAT y coordinación en la evaluación);

5) colaboración con los diferentes estamentos del centro (jefatura de estudios, comisión de coordinación pedagógica, coordinación de tutores, etc.) y

6) coordinación con los servicios educativos y de orientación externos.

Para poder desarrollar estas funciones la composición del Departamento ha de ser multidisciplinar.

\section{Dinamización y potenciación de la acción tutorial}

Para ello, es necesario tener en cuenta los siguientes aspectos:

- Dignificación de la tarea tutorial. Esto se puede lograr a través de: Un reconocimiento real, por parte de la Administración educativa y de su institución escolar. El profesor-tutor ha de sentirse valorado, motivado, preparado e incentivado, destacando su papel como dinamizador de todo este proceso y se han de facilitar las condiciones apropiadas de tiempo, formación, apoyo de los servicios internos y externos, trabajo en equipo y recursos. 
- Criterios adecuados para la selección de tutores. Se han de adoptar criterios pedagógicos y de orientación: Interés y motivación, características personales, tiempo en el centro, compromisos de acompañar al alumnado en su tarea tutorial.

- Formación de tutores. Se le ha de garantizar al profesorado-tutor una formación tutorial adecuada y ha de estar basada en la propia realidad y en las necesidades del profesorado a nivel personal y a nivel de centro (formación para el desarrollo personal y profesional). Finalmente, esta formación ha de ser asumida por la propia Administración, centro educativo y profesorado.

- Las áreas temáticas de la acción tutorial y su transversalidad. La intervención en orientación y acción tutorial se han de centrar en torno a cuatro áreas temáticas:

1) Orientación para el desarrollo de la carrera;

2) Orientación en los procesos de enseñanza-aprendizaje;

3) Orientación para la prevención y el desarrollo;

4) atención a la diversidad.

Estas áreas no aparecen separadas, sino interrelacionadas, tienen una dimensión de transversalidad las unas respecto las otras.

- La acción tutorial como una tarea cooperativa. En la acción tutorial deben colaborar, aunque en diferente grado, distintos agentes: Dentro del centro (Equipo directivo, equipo docente, orientador, profesor-tutor, profesorado, etc.) y fuera del centro (Equipos de orientación externos, servicios sociales, planificación familiar, área de juventud, servicios socio-laborales, centros de formación ocupacional, escuelas-taller, programas de garantía social). Todo ello, dentro de una actitud de respeto, de colaboración y de responsabilidad compartida.

- La necesidad de crear materiales específicos para la tutoría. Se dispone de abundantes obras sobre esta temática a nivel teórico, pero son escasos los materiales que nos plantean experiencias, estrategias y recursos de acción tutorial. El profesor-tutor no dispone de tiempo ni de preparación para elaborar estos materiales.

- Potenciar además de la tutoría grupal, la tutoría individual y con las familias. Se ha de estimular la tutoría individual y se ha de fomentar la implicación de la familia en la acción tutorial.

- Programación de la tutoría en equipo por parte de todos los agentes implicados: Esta se ha de adaptar a las necesidades de los usuarios y se ha de plantear a dos niveles:

1) En vertical, indicando las áreas temáticas a desarrollar en cada nivel educativo;

2) En horizontal, estableciendo una adecuada coordinación entre los diferentes grupos-clase de un mismo curso.

- La orientación y la acción tutorial integrada en el currículum. La acción tutorial ha de ser inherente a la docencia de cada profesor y ha de estar integrada en el currículum. La acción tutorial ha de dar la dimensión formativa y profesionalizadora al currículum. El equipo docente se convierte en el agente natural de la orientación. El programa de estudios constituye la vía natural a través de la cual se afrontan los objetivos de la orientación. 
- Necesidad de una mayor implicación institucional y de la comunidad en la acción tutorial. La orientación y la acción tutorial no se pueden llevar a cabo, si no existe un mínimo de coordinación de la labor tutorial y orientadora del centro y la colaboración de los agentes de la comunidad.

- Coordinación de los diferentes servicios que actúan en la comunidad. Se ha de establecer una adecuada coordinación con los diferentes servicios educativos, sociales, sanitarios, jurídicos y laborales de la población, mediante convenios marco con los responsables de estos servicios.

\section{Presupuestos para la operativización de la propuesta organizativa de la Orientación en España}

Como hemos señalado en otros trabajos (Álvarez González y Rodríguez Espinar, 2000; Marín y Rodríguez Espinar, 2001), la propuesta que se expone a continuación tiene la finalidad, el propósito de concienciar a los responsables de las diferentes CC.AA. de la necesidad de iniciar un debate que permita consensuar unas determinadas medidas de tipo legal (jurídico-administrativo) que faciliten la operativización de la propuesta organizativa de la orientación, que a continuación se explicitan:

\section{Punto de partida}

La concepción de la orientación como un proceso de ayuda y mediación a lo largo de toda la vida de la persona, como elemento facilitador de su desarrollo personal, formativo, social y profesional. Esto va a requerir de una acción coordinada de todos los agentes favorecedores de su desarrollo y de una adecuada organización de la acción orientadora (el marco jurídico-administrativo facilitador y no obstaculizador de la práctica orientadora).

\section{Un reto especial: La atención a la diversidad}

Es uno de los retos más significativos de la nueva ordenación educativa y es, a su vez, el núcleo de la función orientadora desde la perspectiva preventiva y de desarrollo. La atención a la diversidad reclama actuaciones más preventivas que remediales, coordinadas y no ocasionales, organizadas y no arbitrarias, profesionales y no aficionadas (estructura fragmentada y desequilibrada). Esto va a requerir de una auténtica reestructuración que permita la continuidad e intensidad de adecuados tratamientos multidisciplinares y una integración de los servicios educativos y de orientación de la comunidad. Se trata de adoptar un concepto amplio de diversidad, donde tenga cabida la prevención, el desarrollo y la intervención social.

\section{Una apuesta de futuro: los proyectos conjuntos (el partenariado)}

La compleja realidad de la práctica orientadora, la multidisciplinariedad requerida para afrontar intervenciones eficaces (fracaso escolar, toma de decisiones, absentismo, conductas 
antisociales, conflictos, etc.), plantea la necesidad de acciones conjuntas globales entre los orientadores que pertenecen a diferentes instituciones (educación y trabajo; educación y sanidad; trabajo y sanidad, educación y justicia, etc.). Estos proyectos conjuntos van a permitir aunar el conocimiento y los recursos necesarios para afrontar los retos de una eficaz y eficiente acción orientadora.

Ahora bien, no va a resultar nada fácil una acción integradora y multidisciplinar de los distintos servicios, debido a diferencias de tipo organizativo, teóricas y de financiación. Para intentar paliar los efectos negativos de tales diferencias se proponen las siguientes acciones:

- Desarrollo de un plan estratégico con la formalización de contratos y programas específicos de actuación.

- Implicación de los responsables al más alto nivel en la dirección del plan.

- Responsabilidad total en el plan con clara especificación de los agentes y momentos de acción.

- Adopción de mecanismos de coordinación, motivación y evaluación continuada.

- Reafirmación constante del partenariado (no sólo desde los servicios participantes, sino de los destinatarios de los mismos).

Para abordar las diferentes temáticas es necesario que la acción pública se planifique, teniendo en cuenta no sólo los recursos de la Administración, sino el resto de recursos de la sociedad.

\section{Un requisito para la acción: La sectorización}

Para una adecuada planificación de la acción orientadora se han de tener en cuenta los siguientes aspectos:

- Delimitar las unidades territoriales significativas. El escenario ideal es el Municipio. Es el espacio geográfico idóneo para la planificación operativa de la acción orientadora. Esto va a requerir la creación de la comisión municipal de orientación que garantizará la necesaria elaboración del Plan Municipal.

- Desde la perspectiva exclusivamente educativa, los centros de Infantil, Primaria y Secundaria asumirán el referente de actuación, junto con otros centros educativos (adultos, formación ocupacional), el primer nivel organizativo de planificación y coordinación de la actividad orientadora, en consonancia con el Plan Municipal. El programa de orientación de centro y el Departamento de orientación constituyen la célula básica de actuación.

- La especialización de los equipos de apoyo que han de cambiar el criterio territorial (ámbito territorial geográfico) por el criterio de especialización (roles y funciones asignados en virtud de las necesidades detectadas y de las competencias requeridas).

\section{Una consecuencia: Flexible organización de los recursos humanos}

Estos recursos al servicio de la acción orientadora no pueden organizarse de modo rígido y definitivo. Se apuntan tres consideraciones: 
1) Reordenación de los recursos del sector;

2) Articulación de la permanencia o mobilidad intrasector e intersector $y$

3) Estratificación organizativa de los recursos humanos.

\section{Un corolario: La evaluación de la orientación}

Se ha de aunar la rendición de cuentas y la mejora de la propia práctica orientadora. Estratégicamente, se ha de abogar por una evaluación orientada al fortalecimiento de los propios agentes de la orientación en un contexto de interrelación de agentes y servicios (empowerment evaluation). Este tipo de evaluación ayuda a los agentes del programa a evaluarse ellos mismos y su programa para mejorar la práctica y favorecer la autodeterminación y la colaboración. Los participantes del programa conducen su propia evaluación, actúan como facilitadores.

\section{Una necesidad: Ley específica de orientación}

Lo anteriormente expuesto sería difícil ponerlo en práctica con el actual marco jurídico-administrativo. Sólo la existencia de una norma de rango y cobertura nacional podría permitir las nuevas propuestas organizativas y estructurales de la orientación. Una ley de orientación escolar y profesional permitiría definir las líneas maestras de un plan estratégico de nivel nacional como referente de los planes o programas propios de cada Comunidad Autónoma (dimensión nacional de la orientación). Esta medida organizativa facilitaría: la cooperación interterritorial, el adecuado nivel de calidad de la prestación orientadora, la integración de recursos, la definición de las competencias profesionales, la coordinación entre los diferentes servicios educativos, laborales, sanitarios, sociales, etc.

\section{Otra necesidad: El asociacionismo profesional}

La potenciación del asociacionismo profesional como facilitador de: la comunicación y la cooperación entre los asociados, el desarrollo profesional a través de la formación continuada, la promoción y la responsabilidad entre las instituciones y la neutralidad ante posibles injerencias de los Gobiernos, empresarios, institución educativa (Álvarez González, 1998b).

\section{Conclusiones y prospectiva}

Este estudio nos ha permitido hacer un análisis exhaustivo de los sistemas de orientación en las diferentes Comunidades Autónomas. Este análisis nos ha aportado las siguientes conclusiones:

- Los Sistemas de orientación en las distintas Comunidades Autónomas responden a un único modelo. 
- En la mayoría de las Comunidades Autónomas se ha adulterado el modelo original que contempla los tres niveles de intervención en Infantil, Primaria y Secundaria.

- Hay una tendencia a reforzar los servicios internos.

- El modelo de orientación aparece claramente definido a nivel organizativo, no podemos decir lo mismo a nivel funcional.

- Se está haciendo una intervención bastante fragmentaria y con una cierta discontinuidad. Se produce una falta de coordinación.

- No se dispone de un modelo integrador de formación para todos los implicados en la acción tutorial.

Estas conclusiones nos confirman que estamos en un momento crítico, de cambio de Sistema Educativo y, al mismo tiempo, en una fase de clarificación, implantación y ajuste del modelo de orientación, donde las Comunidades Autónomas con nuevas transferencias en Educación no han tenido la valentía de asumir dicho modelo como planteaba la LOGSE, con tres niveles de intervención no sólo en Secundaria, sino también en Infantil y Primaria.

Para la plena implantación del modelo de orientación sería imprescindible hacer hincapié en los siguientes aspectos:

- Desarrollar, de forma plena, un modelo organizativo y funcional de la orientación, basado en tres niveles de intervención (tutoría, Departamento de orientación y Equipos externos), teniendo en cuenta las peculiaridades de cada Autonomía.

- Los Equipos externos han de disponer de una mayor especialización e interdisciplinariedad y han de actuar de forma coordinada con el resto de servicios de la Comunidad (Servicios Sociales, Sanitarios, Jurídicos, Socio-comunitarios y Ocupacionales).

- Implantar los Departamentos de Orientación en los centros de Infantil y Primaria y consolidarlos en Secundaria.

- Dinamizar y potenciar la acción tutorial.

- La necesidad de iniciar un debate, por parte de los responsables de las diferentes Comunidades Autónomas, que permitan consensuar unas determinadas medidas de tipo legal que faciliten la puesta en marcha de la orientación. Estas medidas se podrían concretar en:

- La concepción de la orientación como un proceso de ayuda y mediación a lo largo de la vida.

- La atención a la diversidad reclama actuaciones más preventivas, coordinadas, organizadas y profesionales.

- La compleja realidad de la práctica orientadora plantea la necesidad de acciones conjuntas de las diferentes instituciones y servicios (Desarrollo de un plan estratégico).

- El municipio es el espacio geográfico ideal para la operativización de la acción orientadora (Plan municipal).

- Se ha de afrontar la evaluación para la mejora de la propia práctica orientadora.

- Una Ley de Orientación Escolar y Profesional permitiría definir las líneas maestras de un plan estratégico a nivel nacional, respetando las peculiaridades de cada Comunidad Autónoma (Dimensión nacional de la orientación). 
- La potenciación del asociacionismo profesional como facilitador del desarrollo profesional de los orientadores.

\section{Referencias bibliográficas}

Álvarez González, M. (1994). El modelo organizativo y funcional en el MEC. V Jornades sobre Orientació psicopedagógica i atenció a la diversitat. ACOEP. Barcelona.

Álvarez González, M. (1995). Orientación Profesional. Barcelona: Cedecs.

Álvarez González, M. (1998a). "La orientación educativa, planteamientos y necesidades ante la implantación de la LOGSE". En J. Garcia y J. Cimas (coords.), La orientación educativa y la atención a la diversidad en el marco de Castilla-la Mancha, Toledo (pp. 35-62). Confederación de APAS "Miguel de Cervantes. Castilla la Mancha.

Álvarez González, M. (1998b). "La orientación psicopedagógica en Europa”. En R. Bisquerra (coord.), Modelos de orientación e intervención psicopedagógica (pp. 219-232). Barcelona: Praxis.

Álvarez González, M. y Rodríguez Espinar, S. (2000). "Cambios socioeducativos y orientación en el siglo XXI: Nuevas estructuras, roles y funciones". En AAVV. Hacia el tercer milenio: Cambio educativo y educación para el cambio. XI Congreso Nacional y Iberoamericano de Pedagogía (pp. 637-686). Madrid: Sociedad Española de Pedagogía. Tomo I.

Benavent, J. A. (1996). La orientación psicopedagógica en España: Desde sus orígenes hasta 1939. V. I. Valencia: Promolibro.

Benavent, J. A. (2000). La orientación psicopedagógica en España. Desde 1939 hasta la Ley General de Educación. V. II. Valencia: Promolibro.

Benavent, J. A y Fossati, R. (1998a). "El modelo del MEC". En R. Bisquerra (coord.), Modelos de orientación e intervención psicopedagógica (pp. 133-139). Barcelona: Praxis.

Benavent, J. A. y Fossati, R. (1998b). "Los equipos de orientación educativa y psicopedagógica". En R. Bisquerra (coord.), Modelos de orientación e intervención psicopedagógica (pp. 193-207). Barcelona: Praxis.

Carrión, J. J. y Fernández, J. (1999). “Orientación y legislación: posibilidades y límites para reorientar la acción psicopedagógica”. En J. Fernández (coord.), Acción psicopedagógica en Educación Secundaria: Reorientando la orientación (pp. 121-146). Málaga: Aljibe.

Coll, C. (1998). Rol, perfil i funcions del profesors de Psicología a lènsenyament Secundari a Catalunya. Col.legi oficial de psicólegs de Catalunya i Col.legi Oficial de Doctors i Llicenciats en Filosofia i Lletres en Ciéncies de Catalunya. Barcelona.

Del Valle, A (1987). El modelo de Sevicios Sociales en el Estado de las Autonomías. Madrid: Fundación IESA.

Díaz Allué, M. T. (1989). La orientación y la Reforma de las Enseñanzas. I Seminario Regional de orientación educativa. Murcia

Díaz Allué, M. T. y otros (1997). “Orientación en Educación Secundaria. Situación actual y perspectiva". Revista de Investigación Educativa, 4 (15), 45-66.

García Yagüe, J. (1978). "Posibilidades y límites de la orientación escolar en España”. Patio de Escuelas, 2, 13-38.

Lázaro, A, Asensi, J. y González, M. (1982). "El desarrollo de la orientación institucional en España”. Revista de Educación, 270, pp. 159-182.

Marín, M. A. y Rodríguez Espinar, S. (2001). "Prospectivas del diagnóstico y la orientación". Revista de Investigación Educativa, V. 19, nº 2, pp. 315-362. 
Martín, E. (1999). "La intervención psicopedagógica en el ámbito de gestión del Ministerio de Educación y Cultura: La historia de un difícil pero imprescindible factor de calidad". Infancia y Aprendizaje, 87, pp. 27-45.

Mateo, J. (Coord.)., Álvarez González, M., Casanovas, J., Sans, T. y Hernando, J. C. (2002). "Debat sobre el sistema educativu català. Conclusions i propostes d'avaluació d'aprenentags i orientació. En Generalitat de Catalunya, Departament d'Ensenyament. Consell Superior d'Avaluació del Sistema Educativo". Conferencia Nacional d'Educació. Servei de Difusió y publicacions.

MEC (1987). Proyecto para la Reforma de la Enseñanza. Madrid: publicaciones MEC.

MEC (1989). Libro Blanco para la Reforma del Sistema Educativo. Madrid.

MEC (1990). Ley Orgánica de Ordenación General del Sistema Educativo. LOGSE.

MEC (1990). La Orientación Educativa y la Intervención Psicopedagógica: Publicaciones MEC.

MEC (1992). Orientación y Tutoría. Madrid: Publicaciones MEC.

MEC (2002). Ley Orgánica de Calidad de la Educación (LOCE).

O.M. del 9 de Diciembre de 1992 donde se presenta la estructura y funciones de los Equipos de Orientación Educativa y Psicopedagogía.

Circular de la Dirección General de Renovación Pedagógica del 27 de Julio de 1993 por la que se dictan instrucciones sobre el plan de actividades de los Departamentos de Orientación de los Institutos de Educación Secundaria.

Rodríguez Espinar, S. (1995). "Un reto profesional: la calidad en la investigación orientadora”. En R. Sanz, F., Castallano, y J. A. Delgado (Eds.), Tutoría y Orientación (pp. 119-135). Barcelona: Cedecs.

Rodríguez Espinar, S. (1998). "La orientación ante el reto de nuevos contextos y necesidades". En X de Salvador y Ma. L. Rodicio (coords.), Simposium sobre orientación: ¿para onde camiña a orientación? (pp. 19-37). A Coruña: Servicio de publicaciones Universidade Da Coruña.

Rodríguez Moreno, M. L. (1998). Orientación Profesional. I Teoría. Madrid: ARIEL.

Rodríguez moreno, M. L. (2002). "La orientación escolar y profesional en el sistema educativo español”. En M. Álvarez González y R. Bisquerra (coords.), Manual de Orientación y Tutoría (pp. 28/1-28/22). Barcelona: Praxis.

Sanz, R. (1995). "Las funciones de la Orientación en el marco de la LOGSE. Perspectivas de futuro". En R. Sanz, F. Castellano y J. A. Delgado (coords.), Tutoría y orientación (pp. 9-23). Barcelona: Cedecs.

Sanz, R. (1999). Los Departamentos de Orientación en Educación Secundaria. Roles y funciones. Barcelona: Cedecs.

Sanz, R. (2001). Orientación Psicopedagógica y calidad educativa. Madrid: Pirámide.

Sanz, R. y Sobrado, L. (1998). "Roles y funciones de los orientadores". Revista de Investigación Educativa, V. 16, n' 2, 25-57.

Sobrado, L. (1998). "Servicios externos de orientación a los centros educativos: los modelos de las Comunidades Autónomas". Revista Española de Orientación Psicopedagógica, V. 9, n 15, pp. 109-132.

Sobrado, L. (1999). "Servicios Internos de Orientación en el Sistema Educativo Español”. Revista Española de Orientación Psicopedagógica, V. 10, n 17, 207-226.

Sobrado, L. y otros (1998). "Los modelos de las Comunidades Autónomas". En R. Bisquerra (coord.), Modelos de orientación e intervención psicopedagógica (pp. 209-218). Barcelona: Praxis. 
Santos, M. A. (1995). "Piedras en el camino. Dificultades de los Departamentos de Orientación en los Centros". En J. Fernández (coord.), El trabajo docente y psicopedagógico en Educación Secundaria (pp. 269-283). Málaga: Aljibe.

Tarrego, J. C. (1998). El rol, perfil y funciones del profesor de psicología y pedagogía en la educación secundaria: Reflexiones para el análisis de la situación en la comunidad de Madrid. $1^{\text {as }}$ Jornades de reflexió i formació del professor de psicología i pedagogía. Col.legi oficial de psicólogs de Catalunya i Col.legi Oficial de Doctors i Llicenciats en Filosofía y lletres en Ciéncies de Catalunya. Barcelona.

Fecha de recepción: 26-11-03

Fecha de revisión: 10-03-05

Fecha de aceptación: 20-04-05 